\title{
Weighted Residual Method for the System of Nonlinear Volterra Integral Equations of the Second Kind Using an Exponential Function
}

\author{
Chinar Sh. Ahmed \\ Department of Mathematics /College of Education / University of Garmian \\ chinar.shahab@garmian.edu.krd
}

Article history: Received17,March,2021, Accepted 11 April 2021, Published in July 2021.

Doi: 10.30526/34.3.2682

\begin{abstract}
The numerical resolve nonlinear system of Volterra integral equation of the second kind (NLSVIEK2) has been considered. The exponential function is used as the base function of the collocation method to approximate the resolve of the problem. Arithmetic epitome are performed which have already been solved by weighted residual manner, Taylor manner and block- by- block(2, 3, 5).
\end{abstract}

Keywords: Collocation method, system of nonlinear Volterra integral equation, exponential function.

\section{Introduction}

Solving the system of (linear and nonlinear) VIE of the second kind, many methods with enough accuracy and efficiency have already been used by many researches [1 - 9].

We use widened manner to approximate the resolve of the NLVIEK2 since one of its uses is to exchange intricate careers by some unpretentious careers so that integrated processes can be more unpretentious uttered.

The approximate manners provide analytical practice for obtaining resolves in the form of careers, which are close in some sense to the accurately resolve of the given problem [10].

A collocation manner $(\mathrm{CM})$ has been used for solving integral and integro-differential equations by many authors and researchers $[3,5,6,8,10,11,12,13,14,15,16,17]$.

The nonlinear SVIEK2 is defined as follows:

$$
\varphi_{\ell}(\eta)=\phi_{\ell}(\eta)+\int_{a}^{\eta} k_{\ell}\left(\eta, \lambda, \varphi_{1}(\lambda), \ldots, \varphi_{\gamma}(\lambda)\right) d \lambda ; \eta \in H=[a, b], \ell=1(1) \gamma
$$


where $\gamma \in \Omega$ (denotes $\ell=1(1) \gamma, k_{\ell}$ and $; \phi_{\ell} \in C[a, b] ; \phi_{\ell}, \ell=1(1) \gamma$ natural number) $\Omega$ given continuous careers on $\{(\eta, \lambda): a \leq \lambda \leq \eta \leq b\}$, while $\varphi_{\ell}(\eta), \quad \ell=1(1) \gamma$ are the unknown careers.

\section{Resolve a System of Non-linear VIEK2 by a Collocation Manner:}

A collocation manner is based on approximating the resolve $\varphi_{\ell}(\eta), \ell=1(1) \gamma$ by a partial sum

$$
S_{\ell \Omega}(\eta)=\sum_{\alpha=0}^{\Omega} \theta_{\ell \alpha} \Phi_{\alpha}(\eta) \quad, \ell=1(1) \gamma
$$

where $\Phi_{\Omega}(\eta)$ naturally be choosing linearly independent ( in this work, we choose $\left.\Phi_{\Omega}(\eta)=(\exp (\eta))^{\Omega}, \alpha=0(1) \Omega\right)$ is a weighted function and its widening coefficients $\theta_{\ell 0}, \theta_{\ell 1}$ , ..., $\theta_{\ell \Omega}, \ell=1(1) \gamma$ are to be determined uniquely $[19,20]$.

So by putting equation (2) in equation (1) for $\varphi_{\ell}(\eta), \ell=1(1) \gamma$ we get the residual equation $E_{\ell \Omega}\left(\eta, \theta_{\ell 0}, \theta_{\ell 1}, \ldots, \theta_{\ell \Omega}\right)$ such that:

$$
E_{\ell \Omega}\left(\eta, \theta_{\ell \alpha} ; \alpha=\overline{0, \Omega}\right)=S_{\ell \Omega}(\eta)-\int_{a}^{x} K_{\ell}\left(\eta, \lambda, S_{1 \Omega}(\eta), S_{2 \Omega}(\eta), \ldots, S_{\curlywedge \Omega}(\eta)\right) d \lambda-\phi_{\ell}(\eta), \ell=1(1) \gamma
$$

The considerations $\theta_{\ell 0}, \theta_{\ell 1}, \ldots, \theta_{\ell \Omega}, \ell=1(1) \gamma$ must establish by forcing that the residual $E_{\ell \Omega}\left(\eta, \theta_{\ell 0}, \theta_{\ell 1}, \ldots, \theta_{\ell \Omega}\right)$ disappears at a certain set of $\Omega+1$ numbers in the ambit $D$. So by choosing $\Omega+1$ distinct nodes $\eta_{0}, \eta_{1}, \ldots, \eta_{\Omega} \in D$ substituting in equation (3) with the approximate resolve in equation (2), we get $\mu=\gamma \times(\Omega+1)$ simultaneous equations so

$$
\begin{aligned}
& E_{\ell \Omega}\left(\eta_{\beta}, \theta_{\ell 0}, \theta_{\ell 1}, \ldots, \theta_{\ell \Omega}\right)=0 \\
& \sum_{\alpha=0}^{\Omega} \theta_{\ell \Omega}\left(\exp \left(\eta_{\beta}\right)\right)^{\alpha}-\phi_{\ell}\left(\eta_{\beta}\right)-\int_{a}^{\eta_{\beta}} K_{\ell}\left(\eta_{\beta}, \lambda, \sum_{\alpha=0}^{\Omega} \theta_{1 \alpha}(\exp (\lambda))^{\alpha}, \sum_{\alpha=0}^{\Omega} \theta_{2 \alpha}(\exp (\lambda))^{\alpha}, \ldots, \sum_{k=0}^{\Omega} \theta_{\gamma \alpha}(\exp (\lambda))^{\alpha}\right) d \lambda=0 \\
& \ell=1(1) \gamma
\end{aligned}
$$

where $\eta_{\beta}=\beta h, \beta=0(1) \mu$ and $h$ is to be chosen.

Equation (4) leads to the following non-linear system of equations

$$
\begin{aligned}
& \sum_{\alpha=0}^{\Omega} \theta_{\ell \Omega}\left(\exp \left(\eta_{\beta}\right)\right)^{\alpha}-\int_{a}^{\eta_{\beta}} K_{\ell}\left(\eta_{\beta}, \lambda, \sum_{\alpha=0}^{\Omega} \theta_{1 \alpha}(\exp (\lambda))^{\alpha}, \sum_{\alpha=0}^{\Omega} \theta_{2 \alpha}(\exp (\lambda))^{\alpha}, \ldots, \sum_{k=0}^{\Omega} \theta_{\gamma \alpha}(\exp (\lambda))^{\alpha}\right) d \lambda=-\phi_{\ell}\left(\eta_{\beta}\right) \\
& \ell=1(1) \gamma, \beta=1(1) \mu .
\end{aligned}
$$

Here, we need to determine $\mu$ coefficients $\theta_{\vartheta q}, \vartheta=1(1) \gamma, q=0(1) \Omega$. We construct $\mu \times \mu$ system of equations from equation (5) to located coefficient $\theta_{\ell 0}, \theta_{\ell 1}, \theta_{\ell 2}, \ldots, \theta_{\ell \Omega}$, and substitute the values of the coefficients into the equation (2), we obtain the approximate resolve equation (1). 
Ibn Al-Haitham Jour. for Pure \& Appl. Sci. 34(3)2021

\section{Illustrative Epitome:}

To illustrate the application of the collocation method we use some examples of nonlinear system of VIEK2's in the following epitomes:

\section{Epitome 3.1:}

The nonlinear system of VIEK2

$$
\begin{aligned}
& \varphi_{1}(\eta)=\eta-\eta^{2}+\int_{0}^{\eta}\left(\varphi_{1}(\lambda)+\varphi_{2}(\lambda)\right) d \lambda \\
& \varphi_{2}(\eta)=\eta-\frac{1}{2} \eta^{2}-\frac{1}{3} \eta^{3}+\int_{0}^{\eta}\left(\varphi_{1}^{2}(\lambda)+\varphi_{2}(\lambda)\right) d \lambda
\end{aligned}
$$

have the following accurate resolves $\varphi_{1}(\eta)=\eta, \varphi_{2}(\eta)=\eta$

\section{Resolve:}

We have the accurate resolve $\varphi_{1}(\eta)=\eta, \varphi_{2}(\eta)=\eta$. Table 1 shows the estimate error of the current manner (Manner 1) with Taylor manner (Manner 2) [2], weighted residual manner using the polynomial function (Manner 3) [5] and the Block-by-block manner (Manner 4) $[4,8]$ for $\Omega=2,3$.

\begin{tabular}{|c|c|c|c|c|c|}
\hline \multirow{2}{*}{\multicolumn{2}{|c|}{ Manners }} & \multicolumn{2}{|c|}{$\Omega=2$} & \multicolumn{2}{|c|}{$\Omega=3$} \\
\hline & & $\varphi_{1}(\eta)$ & $\varphi_{2}(\eta)$ & $\varphi_{1}(\eta)$ & $\varphi_{2}(\eta)$ \\
\hline \multicolumn{2}{|c|}{ Manner 1} & $91833 \times 10^{-2}$ & $4.7934 \times 10^{-3}$ & $5.7732^{\times 10^{-2}}$ & $8.6877 \times 10^{-3}$ \\
\hline \multicolumn{2}{|c|}{ Manner 2} & 0 & 0 & 0 & 0 \\
\hline \multicolumn{2}{|c|}{ Manner 3} & 0 & 0 & 0 & 0 \\
\hline \multirow{3}{*}{ Manner 4} & MBLM2 & 0 & 0 & 0 & 0 \\
\hline & MBLM3 & $7.6115 \times 10^{-2}$ & $7.3900 \times 10^{-2}$ & $2.5163 \times 10^{-3}$ & $1.2612 \times 10^{-3}$ \\
\hline & MBLM5 & $6.4110 \times 10^{-02}$ & $6.0717 \times 10^{-02}$ & $2.2870 \times 10^{-03}$ & $1.0959 \times 10^{-03}$ \\
\hline
\end{tabular}

Table 1. Comparison of the errors of Epitome 1.

\section{Epitome 3.2:}

$$
\begin{aligned}
& \varphi_{1}(\eta)=\sec (\eta)-\eta+\int_{0}^{\eta}\left(\left(\varphi_{1}(\lambda)\right)^{2}-\left(\varphi_{2}(\lambda)\right)^{2}\right) d \lambda \\
& \varphi_{2}(\eta)=3 \tan (\eta)-\eta-\int_{0}^{\eta}\left(\left(\varphi_{1}(\lambda)\right)^{2}+\left(\varphi_{2}(\lambda)\right)^{2}\right) d \lambda
\end{aligned}
$$

We have the accurate resolve $\varphi_{1}(\eta)=\sec (\eta)$ and $\varphi_{2}(\eta)=\tan (\eta)$. Table 2 shows the estimate error of the current manner (Manner 1) with Taylor manner (Manner 2) [2], weighted residual manner using polynomial function (Manner 3) [5] and the Block-by-block manner (Manner 4) [4, 8] for $\Omega=2,3$. 
Ibn Al-Haitham Jour. for Pure \& Appl. Sci. 34(3)2021

Table 2. Comparison of the errors of Epitome 2.

\begin{tabular}{|c|c|c|c|c|c|}
\hline \multirow{2}{*}{\multicolumn{2}{|c|}{ Manners }} & \multicolumn{2}{|c|}{$\Omega=2$} & \multicolumn{2}{|c|}{$\Omega=3$} \\
\hline & & $\varphi_{1}(\eta)$ & $\varphi_{2}(\eta)$ & $\varphi_{1}(\eta)$ & $\varphi_{2}(\eta)$ \\
\hline \multicolumn{2}{|c|}{ Manner 1} & $5.5729 \times 10^{-3}$ & $2.4367 \times 10^{-2}$ & $8.2236 \times 10^{-2}$ & $4.2749 \times 10^{-3}$ \\
\hline \multicolumn{2}{|c|}{ Manner 2} & $1.8302 \times 10^{-1}$ & $5.2323 \times 10^{-1}$ & $1.8302 \times 10^{-1}$ & $6.8365 \times 10^{-2}$ \\
\hline \multicolumn{2}{|c|}{ Manner 3} & $8.0421 \times 10^{-2}$ & $1.5473 \times 10^{-2}$ & $5.0644 \times 10^{-2}$ & $3.5296^{\times} \times 10^{-3}$ \\
\hline \multirow{3}{*}{ Manner 4} & MBLM2 & $3.4937 \times 10^{-1}$ & $1.0005 \times 10^{-1}$ & $4.6744 \times 10^{-3}$ & $3.9331 \times 10^{-3}$ \\
\hline & MBLM3 & $5.1058 \times 10^{-1}$ & $3.2951 \times 10^{-1}$ & $1.2311 \times 10^{-2}$ & $1.2225 \times 10^{-2}$ \\
\hline & MBLM5 & $1.1904 \times 10^{-02}$ & $6.9640 \times 10^{-02}$ & $3.3693 \times 10^{-03}$ & $4.7832 \times 10^{-03}$ \\
\hline
\end{tabular}

\section{Epitome 3.3:}

Solve a system of non-linear VIEK2's:

$$
\begin{aligned}
& \varphi_{1}(\eta)=\frac{1}{4}-\frac{1}{4} e^{2 \eta}+\int_{0}^{\eta}(\eta-\lambda) \varphi_{2}^{2}(\lambda) d \lambda \\
& \varphi_{2}(\eta)=-\eta e^{\eta}+2 e^{\eta}-1+\int_{0}^{\eta} \lambda e^{-2 \varphi_{1}(\lambda)} d \lambda
\end{aligned}
$$

The exact resolve of this system are $\varphi_{1}(\eta)=-0.5 \eta$ and $\varphi_{2}(\eta)=e^{\eta}$. Table 3 shows the estimate error of current manner (Manner 1) with Taylor manner (Manner 2) [2], weighted residual manner using polynomial function (Manner 3) [5] and the Block-by-block manner (Manner 4) $[4,8]$ for $\Omega=2,3$.

\begin{tabular}{|c|c|c|c|c|c|}
\hline \multirow{2}{*}{\multicolumn{2}{|c|}{ Manners }} & \multicolumn{2}{|c|}{$\Omega=2$} & \multicolumn{2}{|c|}{$\Omega=3$} \\
\hline & & $\varphi_{1}(\eta)$ & $\varphi_{2}(\eta)$ & $\varphi_{1}(\eta)$ & $\varphi_{2}(\eta)$ \\
\hline \multicolumn{2}{|c|}{ Manner 1} & $2.4932 \times 10^{-3}$ & $7.3509 \times 10^{-3}$ & $1.1968 \times 10^{-3}$ & $6.4439 \times 10^{-4}$ \\
\hline \multicolumn{2}{|c|}{ Manner 2} & 0 & 1.1914 & 0 & $8.9917 \times 10^{-2}$ \\
\hline \multicolumn{2}{|c|}{ Manner 3} & $5.2255 \times 10^{-4}$ & $7.9235 \times 10^{-4}$ & $2.2115 \times 10^{-4}$ & $1.0035 \times 10^{-5}$ \\
\hline \multirow{3}{*}{ Manner 4} & MBLM2 & $5.9824 \times 10^{-2}$ & $5.4301 \times 10^{-2}$ & $1.5734 \times 10^{-3}$ & $2.0101 \times 10^{-4}$ \\
\hline & MBLM3 & $7.7723 \times 10^{-3}$ & $2.2726 \times 10^{-2}$ & $6.5937 \times 10^{-5}$ & $8.5190 \times 10^{-4}$ \\
\hline & MBLM5 & $7.8419 \times 10^{-02}$ & $7.8582 \times 10^{-02}$ & $1.7864 \times 10^{-03}$ & $9.7120 \times 10^{-04}$ \\
\hline
\end{tabular}

Table 3. Comparison of the errors of Epitome 3.

\section{Conclusion}

Many authors solved Non-linear system VIEK2 approximately and analytically. It is enough to obtain the approximate resolves. Since the analytical resolve is very complicated, so we proposed the offered manner to find the approximate resolve. In practice, we get the good accuracy if we choose $\mathrm{n}$ sufficiently large $\mathrm{n}$ and a suitable choice of collocation point. We compare the present manner and those from aforementioned manners in order to choose the best manner to get the approximate resolve for the problem of this system where it is difficult to find the accrue resolve. 
Ibn Al-Haitham Jour. for Pure \& Appl. Sci. 34(3)2021

\section{References}

1. Babolian, E.; Biazar, J., Resolve of a System of Nonlinear Volterra Integral Equations of the Second Kind. Far East J. Math. Sci. (FJMS), 2000, 2, 6, , 935-946.

2. Saeed, R., K.; Ahmed, C.S. Approximate Resolve for the System of Nonlinear Volterra Integral Equations of the Second Kind by Taylor Series Expansion. Indian Journal of Natural Sciences, 2019, 9, 53.

3. Saeed, R. K.; Computational Manners for Solving System of Linear Volterra Integral and Integro-Differential Equations. PhD. Thesis, University of Salahaddin, Department of Applied Science, 2006.

4. Saeed, R., K.; Ahmed, C.S. Approximate Resolve for the System of Non-linear Volterra Integral Equations of the Second Kind by using Block-by-block Manner. Australian Journal of Basic and Applied Sciences, 2008, 2, 1, 114-124.

5. Saeed, R., K.; Ahmed, C.S. Approximate Resolve for the System of Nonlinear Volterra Integral Equations of the Second Kind by Weighted Residual Manners. Journal of Kirkuk University-Scientific Studies, 2009, 5, 1.

6. Şahin,N.; Yüzbaşı, Ş.; Gülsu, M. A collocation approach for solving systems of linear Volterra integral equations with variable coefficients. Computers and Mathematics with Applications, 2011, 62, 755-769.

7. Wazwaz, A.-M., The modified decomposition manner for analytic treatment of nonlinear integral equations and systems of non-linear integral equations. International, 2005.

8. Ahmed, Chinar S. Approximate Resolve for the System of Non-linear Volterra Integral Equations of the Second Kind by Five Block-by-block Method. Open Science Repository Mathematics Online, 2013, e70081992. doi:10.7392/openaccess.70081992.

9. Al-Faour, O. M. A.; Saeed R. K. Resolve of a System of Linear Voltera - Integro Differential Equations by Weighted Residual Method. Journal of Al-Nahrain University, 2007, 10, 1, 84-93.

10. Boyed, J. P. Chebyshev and Fourier Spectral Manner; DOVER publications, Inc., Minneola, New York, 2000.

11. Calio, F.; Marchetti , E.; Pavani, R.; Micula, GH. About Some Volterra Problems Solved BY A Particular Spline Colocation. STUDIA UNIV. "BABES,-BOLYAI", MATHEMATICA, Volume XLVIII, 2003, 3. 
Ibn Al-Haitham Jour. for Pure \& Appl. Sci. 34(3)2021

12. Hendi, F.A.; Albugami, A.M. Numerical resolve for Fredholm-Volterra integral equation of the second kind by using collocation and Galerkin methods. Journal of King Saud University (Science), 2010, 22, 37-40.

13. BAZM , S. Resolve of NonLinear Volterra-Hammerstein Integral Equation Using Alternative Legendre Collocation Method. Sahand Communications in Mathematical Analysis (SCMA), 2016, 4, 1, 57-77.

14. Saeed, R., K.; Hassan, J.S., Solving Singular Integral Equations by using Collocation Method. Math. Sci. Lett., 2014, 3, 3, 185-187.

15. Agbolade, O.A. and Anake, T.A. Resolves of First-Order Volterra Type Linear Integro differential Equations by Collocation Method. Journal of Applied Mathematics, Article ID 1510267, 2017, 5 .

16. Shahsavaran, A. Numerical Approach to Solve Second Kind Volterra Integral Equation of Abel Type Using Block-Pulse Careers and Taylor Expansion by Collocation Method. Applied Mathematical Sciences. 2011, 5, 14, 685 - 696.

17. Ghadle , K.P.; Hamoud, A. A. On the Numerical Resolve of Volterra-Fredholm Integral Equations with Exponential Kernal using Chebyshev and Legendre Collocation Methods. Applied Mathematics, Elixir Appl. Math. 2016, 95, 40742-40746.

18. Shahsavaran, A. Numerally Approach to Solve Second Kind Volterra ntegral Equation of Abel Type Using Block-Pulse Careers and Taylor Expansion by Collocation Manner. Applied Mathematical Sciences, 2011, 5, 14, 685 - 696.

19. Delves, L. M. and Walsh, J. Numerally Resolve of Integral Equations; Claredon Press Oxford, 1974.

20. Polyanin, A. D.; Manzhirov, A. V. Handbook of Integral Equations; CRC Press LLC, 1998. 